\title{
Energy-aware scheduling of malleable HPC applications using a Particle Swarm optimised greedy algorithm
}

\author{
Briag Dupont ${ }^{\mathrm{a}, *}$, Nesryne Mejri ${ }^{\mathrm{a}}$, Georges Da Costa ${ }^{\mathrm{b}}$ \\ a University of Luxembourg, Luxembourg \\ ${ }^{\mathrm{b}}$ IRIT Laboratory, University of Toulouse, France
}

\section{A R T I C L E I N F O}

\section{Keywords:}

Energy efficiency

Malleable jobs

Particle Swarm Optimisation

High performance computing

Scheduling

\begin{abstract}
A B S T R A C T
The scheduling of parallel tasks is a topic that has received a lot of attention in recent years, in particular, due to the development of larger HPC clusters. It is regarded as an interesting problem because when combined with performant hardware, it ensures fast and efficient computing. However, it comes with a cost. The growing number of HPC clusters entails a greater global energy consumption which has a clear negative environmental impact. A green solution is thus required to find a compromise between energy-saving and high-performance computing within those clusters. In this paper, we evaluate the use of malleable jobs and idle servers powering off as a way to reduce both jobs mean stretch time and servers average power consumption. Malleable jobs have the particularity that the number of allocated servers can be changed during runtime. We present an energy-aware greedy algorithm with Particle Swarm Optimised parameters as a possible solution to schedule malleable jobs. An in-depth evaluation of the approach is then outlined using results from a simulator that was developed to handle malleable jobs. The results show that the use of malleable tasks can lead to an improved performance in terms of power consumption. We believe that our results open the door for further investigations on using malleable jobs models coupled with the energy-saving aspect.
\end{abstract}

\section{Introduction}

The current world is massively abundant with valuable data. This by itself represents a reason - among others - for the increasing number of High-Performance Computing (HPC) clusters, data centres, and cloud computing services seen in the past few years. This need for constantly processing enormous loads of data requires not only physical means but also software actors capable of using the hardware capabilities to its fullest. These actors should act in an objectiveoriented way when handling data. This is a scheduling problem that is not considered new, neither in applied fields nor in research. Indeed it has been a hot topic for years since it touches various domains such as industrial processes, manufacturing, computing and transportation planning. In most of these applications, it is mainly concerned with how to dispatch a set of processable resources over a finite set of processing resources, in a way that optimises one or several objectives. Unsurprisingly it is the topic of this paper as there is a growing interest in parallel and distributed computing where it is critical to ensure a smooth execution for the distributed applications. Another related matter that rises is energy efficiency, as more HPC clusters and data centres increase the $\mathrm{CO}_{2}$ emission rate. As a matter of fact [1] states that digital technologies account for between $2.5 \%$ to $3.7 \%$ of the global greenhouse gas emissions. Data centres represent $19 \%$ of the total energy required for the production and use of digital devices. The energy consumed by data centres is expected to increase by about $4 \%$ per year. Clearly reducing the energy consumption should be a goal and this issue should be approached from different angles. Moreover it has been shown that servers account for up to $75 \%$ of a data centre energy consumption [2], and within this value, $50 \%$ can be due to idle servers [3]. The total energy consumed is not the only issue, the rate at which energy is consumed (power) must also be considered as it is bounded. For example the US Department of Energy EISC (Exascale Initiative Steering Committee) has set $20 \mathrm{MW}$ as the upper limit for a buildable exascale system [4].

The energy saving problem can be solved from different perspectives, such as the hardware one or the software one. From the software part, a scheduler implementation can play a key role in the energy consumption of a data centre. Usually, there are two main requirements to consider when building a scheduler; fairness and energy consumption. A scheduler is fair when it guarantees that every submitted job has a chance to be executed within a reasonable time. This is known as fair-share mechanisms [5].

\footnotetext{
* Corresponding author.

E-mail address: briag.dupont.001@student.uni.lu (B. Dupont).
} 

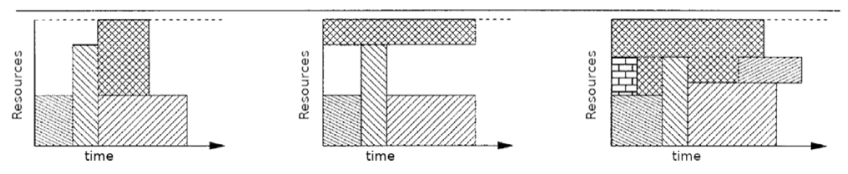

Fig. 1. Scheduling of moldable jobs (left, middle) and of malleable jobs (right) (from [8]).

Hence, the combined cost of a fair, performant and energy-efficient scheduler should be optimised to achieve an efficient use of servers and a lower energy and power consumption within data centres and HPC clusters. In the following section, a brief review of the literature is made. Next, the optimisation problem is formally presented by outlining the objectives and the performance criteria. Afterwards, the used approach is thoroughly explained along with the adopted assumptions and the optimisation process. Following, the experimental setup is described. Lastly, the experimental results are presented and discussed.

\section{Literature review}

Since scheduling parallel jobs is an NP-Hard problem [6] no known algorithm can solve it in polynomial time, and only approximations can be used to find near-optimal solutions. Consequently, the matter has been approached from different perspectives and setups depending on the used scheduling algorithm and the nature of the tasks to perform. Trystram-Mounié et al. [7] described approximations for algorithms taking into account diverse paradigms of jobs; rigid ones, moldable ones, and malleable ones, analysing and comparing the theoretical performance of each paradigm. Rigid jobs have a fixed number of required resources that cannot be changed once a job has been scheduled. Furthermore, if this number is greater than the number of available resources, the job may not be scheduled. Similarly, for moldable jobs, the number of required resources cannot be changed during runtime. However, the number of resources to allocate is defined by the scheduler before the job starts its execution. Lastly, malleable jobs are the most flexible ones since they behave like moldable jobs with the exception that the scheduler can adapt the number of their required resources even during execution [8]. For malleable jobs, the process of changing the number of required resources at runtime is called a reconfiguration. An example depicting malleable jobs and rigid jobs can be seen in Fig. 1.

An approach from literature [9] uses the malleable jobs paradigm because of its flexibility and efficiency. However, assumptions are made about the homogeneity of the cluster, and PTGs (Parallel Task Graphs or Directed Acyclic Graphs) are considered as perfect malleable tasks. These assumptions do not reflect real-life setups. In reality, malleable jobs cannot be perfect, and their reconfiguration necessarily induces overheads such as communication between nodes and time for data to be transferred. Another work [10] addressing cloud computing services focuses more on the optimisation aspect of providing high QoS and the improvement of energy consumption. It also provides a comparison of the Mixed Integer Linear Programming (MILP) approach against the Genetic Algorithm approach for the same objectives.

Finally, cutting-edge simulators such as Batsim [11] or SimGrid [12] do not support the scheduling of malleable jobs. Consequently, researchers are restricted from experimentally investigating potential improvements of performance offered by this paradigm.

\section{Formulation of the problem}

This section formally introduces the optimisation problem, that is given a set of malleable jobs, the goal is to find a scheduling strategy that optimises the performance and the power consumption by respectively using job reconfigurations and turning off idle servers. We define the following useful notions:
Table 1

Reference values for servers power consumption.

\begin{tabular}{ll}
\hline Measure & Value \\
\hline Power idle & $95.00 \mathrm{~W}$ \\
Power while computing & $190.74 \mathrm{~W}$ \\
Power while turning on & $125.17 \mathrm{~W}$ \\
Power while turning off & $101.00 \mathrm{~W}$ \\
Power while off & $9.75 \mathrm{~W}$ \\
Time to turn on & $151.52 \mathrm{~s}$ \\
Time to turn off & $6.10 \mathrm{~s}$
\end{tabular}

- A job is a set of identical parallel tasks submitted at the same time and that can be run on $n$ distinct servers.

- The submission time is the time at which a job enters the queue of the scheduler.

- The speed-up factor $\alpha$, is a real number between 0.5 and 1 indicating how parallelisable the computations within a job are.

- The mass corresponds to execution time of the job if it were to be run on a single server. In a rigid job context, the mass is directly related to the required time to complete the job, as it would be run over a fixed number of servers.

- The makespan is the time needed to complete a job once it starts executing. In a malleable job context, the completion time of a job also depends on factors such as the number of used servers $n$, the speed-up factor $\alpha$ and whether the job has been reconfigured. For a constant number of servers $n$ it is defined similarly as in [13]:

makespan $=\frac{\text { mass }}{n^{\alpha}}$

- The data $D$ is the amount of data to transfer from $n$ to $m$ servers in case a reconfiguration takes place.

- The reconfiguration time $T_{n \rightarrow m}$ is the time needed to transfer data from $n$ to $m$ servers. It is given by:

$T_{n \rightarrow m}= \begin{cases}\frac{D}{n}\left(\left\lceil\frac{n}{m}\right\rceil-1\right) & \text { if } n \geq m \\ \frac{D}{m}\left(\left\lceil\frac{m}{n}\right\rceil-1\right) & \text { if } n \leq m\end{cases}$

\subsection{Criteria}

The goal of this study is to reduce the average rate of energy consumption or power without compromising the fairness of the scheduler.

Classically, the objective of the scheduler is to minimise either the total makespan (the time to complete all the given jobs) or the mean or maximum stretch time. Due to the distributed aspect of the problem, the stretch time is potentially a more beneficial metric than the makespan, as it takes into account the time a job spends waiting in the queue. In a rigid job context, the stretch time is defined as the ratio of the difference between the termination time and the submission time to the actual job execution time. As outlined earlier, the execution time of malleable jobs can vary as it depends on the number of used servers as well as whether the job has been reconfigured. So the definition of the stretch time is adapted to:

stretch time $=\frac{\text { termination time }- \text { submission time }}{\text { mass }}$

The other criterion to take into account is the power consumption of all the servers. Realistic energy consumption approximations of the different server states were used in this study and are shown in Table 1. These values correspond to measurements made on the Tarus Grid'5000 cluster which was composed of 16 Dell PowerEdge R720 nodes each with two Intel Xeon E5-2630 [14]. It can be easily shown that saving energy by turning off a server is only profitable when the shutdown duration is of at least $260 \mathrm{~s}$. This implies that a cycle of turning off, staying off for the minimum span of $260 \mathrm{~s}$, and restarting a server lasts for about $362 \mathrm{~s}$. In the model, the minimum allowed duration for a power-off is $362 \mathrm{~s}$. 
As a second metric, the normalised mean power consumption is considered. It is defined as the mean power consumption divided by the power consumption of an idle server.

normalised mean power $=\frac{\text { mean power }}{\text { idle server power }}$

\subsection{Objective}

Under the following scheduling constraints:

- A job must eventually be scheduled and completed.

- No two jobs can be scheduled at the same time on the same servers.

- A job can only be scheduled or reconfigured on servers that are powered on and idle.

The goal is to minimise the combined cost of the mean stretch time and the normalised mean power consumption. Therefore the objective function can be formalised as an energy-delay-product (EDP), which is a metric that had been used in [15], and that expresses the trade-off between the performance and the power consumption of our model. Thus the objective function is expressed as:

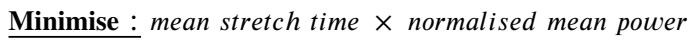

\section{Optimisation strategy}

This section presents the adopted approach for minimising the objective function under the previously mentioned constraints.

\subsection{Assumptions}

The following assumptions were made when implementing the simulation environment supporting malleable jobs.

- The user submits a job request and the scheduler handles it.

- The attributes of a job request are the submission time, the speedup factor $\alpha$, the mass, the minimum and the maximum number of servers required to run the job. All these parameters are considered known to the scheduler.

- The data amount $D$ of a job can be queried by the scheduler at any time. This is a reasonable assumption as in reality, it should be possible to know how much data a program is using at a given time.

- There is an upper limit $D_{\max }$ to the amount of data a job can have, and this limit is known to the scheduler.

- The scheduler has no knowledge about any future states, it only knows about the current and past states.

- The mass submitted by the user is only an estimate. Hence, the scheduler cannot use it to make exact predictions about the termination time.

\subsection{Greedy algorithm}

Adding job reconfigurations and server power-offs to the capabilities of the scheduler increases the complexity of the scheduling problem. Therefore the selected approach to tackling the problem is to implement a greedy algorithm and use a meta-heuristic to tune its parameters.

Greedy algorithms are heuristic-based algorithms that always take the best immediate, or local, solution while finding an answer. Greedy algorithms find the overall or globally optimal solution for some optimisation problems but may find less-than-optimal solutions for some instances of other problems [16]. The implementation steps are described in Algorithm 1

The scheduler's implementation is based on three main parts. The first one prioritises the scheduling of the jobs in the queue per order of time submission. Once the queue is empty and there are still some

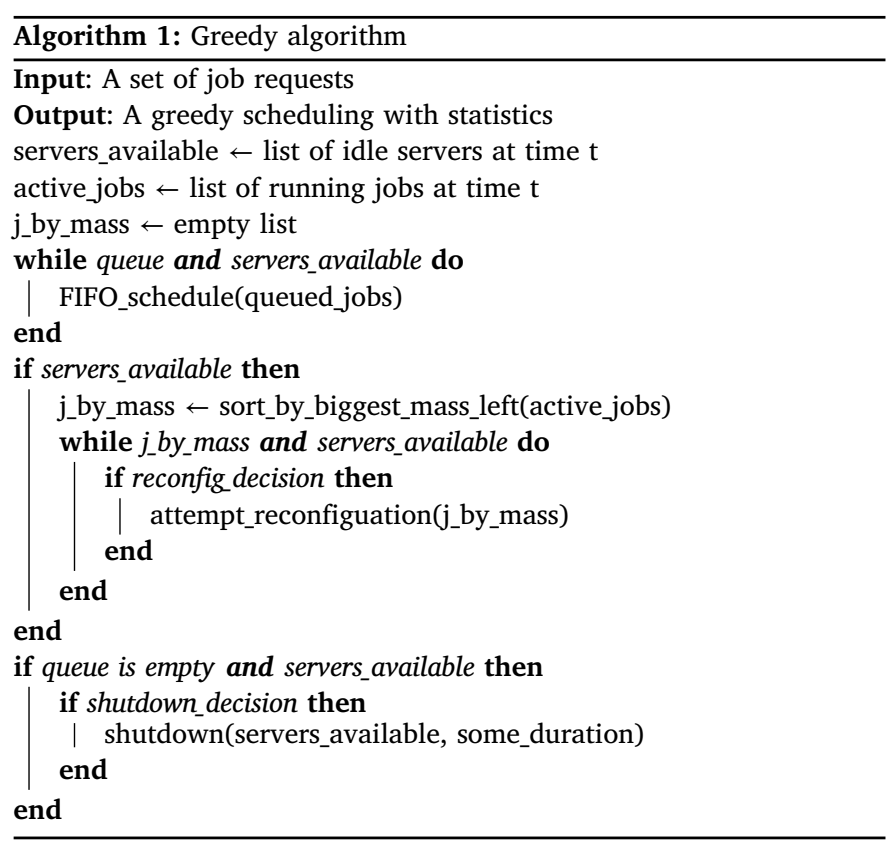

idle servers, the scheduler will try to reconfigure the running jobs by prioritising the ones with the highest remaining mass. The third part takes place when the queue is empty, and there is no possibility of reconfiguring any of the running jobs, at that moment, the scheduler will shut down the unused servers. Again the scheduler's main challenge is that it has no knowledge about the submission time of the upcoming jobs nor their parameters. Consequently, it cannot estimate in advance whether a reconfiguration would actually improve the performance as the servers that will be used for the reconfiguration may be required at any time for a new job. For a similar reason, shutting down idle servers may not always improve power consumption. Hence, in the next two sections, an explanation of the decision processes mentioned as reconfigdecision and shutdown_decision in Algorithm 1 is presented.

\subsubsection{Handling reconfigurations}

Finding a good representation of the decision-making process of the reconfigurations is not a trivial problem. Therefore, three inequalities are introduced as candidates to model the condition at which a reconfiguration should take place. The first condition is considered the most basic one. It takes into account the main factors that are assumed to directly impact the outcome of reconfiguration: that is the number of used servers and the speed-up factor $\alpha$ of the job. It is also considered as the least complex as it introduces only three parameters. It is used later in the report within the setup Swarm1. The common attributes to the three conditions are: $n_{\text {new }}$ which is the new number of servers that a job would be running on after a reconfiguration, $n_{\max }$ is the maximum number of servers a job can be assigned to (specified in the job request), and the speed-up factor $\alpha$. Consequently, the condition 1 is met if:

$\left(\frac{n_{\text {new }}}{n_{\max }}\right)^{w_{n}} \times \alpha^{w_{\alpha}} \times s_{\text {reconfig }}>0.5$

where $w_{n}$ is a weight between 0 and 1 and that is given to the ratio $\frac{n_{\text {new }}}{n_{\max }}$. The speed-up factor $\alpha$ also carries a weight $w_{\alpha} \cdot s_{\text {reconfig }}$ is a scaling factor between 0 and 1 .

The second condition is paired with the setup Swarm2. It is slightly more complex as it introduces an extra parameter $w_{D}$ that needs to be tuned. The idea is that since the duration of a reconfiguration is impacted by the amount of data to transfer between the servers, it would be fair to embed such amount in the decision-making process. 
In that way, a reconfiguration takes place if the following condition2 is met if:

$\left(\frac{n_{\text {new }}}{n_{\max }}\right)^{w_{n}} \times \alpha^{w_{\alpha}} \times\left(\frac{D}{D_{\max }}\right)^{w_{d}} \times s_{\text {reconfig }}>0.5$

where $D$ is the amount of data of the job, and $D_{\max }$ is the maximum amount of data any job can have. This ratio carries a weight $w_{D}$ ranging from 0 to 1 , and that should be also tuned.

Finally condition 3 introduces a bias term instead of the scaling factor $s_{\text {reconfig }}$ which is in the range of -0.5 and 0.5 . It also relies on the tanh function and the previously mentioned parameters to decide whether a reconfiguration should be performed. It is used in the setup Swarm3, and is met if:

$\tanh \left(\left(\frac{n_{\text {new }}}{n_{\max }}\right)^{w_{n}} \times \alpha^{w_{\alpha}} \times\left(\frac{D}{D_{\max }}\right)^{w_{d}}+\right.$ bias $)>0.5$

For condition 1 and condition 2 , the value of 0.5 in each of the conditions is arbitrary, other values were tested and it did not seem to affect significantly the results. For condition $3,0.5$ was chosen over 0 to push the decision process towards using reconfigurations more often as the tanh function is not symmetrical around 0.5.

\subsubsection{Handling power-offs}

For power-offs, it is assumed that the main factor that affects whether it is beneficial to shut down the idle servers is the ratio of available servers to the total number of servers. This decision, referred to as shutdown_decision in Algorithm 1, is based on the following condition:

$\left(\frac{n_{a v}}{n_{\text {tot }}}\right)^{w_{\text {off }}} \times s_{\text {off }}>0.5$

where $n_{a v}$ is the number of available servers, $n_{t o t}$ the total number of servers, $w_{\text {off }}$ a weight between 0 and 1 that reflects the importance of this ratio when making the shutdown decision and $s_{o f f}$ is a scaling factor on the previous ratio. Additionally, instead of always powering off the servers for the same constant amount of time, two different shutdown durations are proposed; $t_{1 \text { off }}$ and $t_{2 \text { off } f}$. Indeed, the scheduler does not know in advance whether the shutdown will be beneficial, so a probability $p_{t_{1} \text { off }}$ for choosing $t_{1 \text { off } f}$ over $t_{2 \text { off }}$ is introduced.

\subsubsection{Greedy algorithm parameters}

The decision process is thus influenced by the combination of the previously mentioned parameters from the shutdown process and the chosen condition of the job reconfiguration. The weights and scaling factors range between 0 and 1 . The bias term range is between -0.5 and 0.5 . The powering off times are only constrained by the $362 \mathrm{~s}$ value that is the minimum duration that guarantees energy saving. Thus during the optimisation process, either eight or nine parameters need to be tuned. A summary of these parameters can be found in Table 2 . Additionally, it is worth mentioning that when tests were run, it was more challenging to find a condition that models the decision-making process of the reconfigurations than finding a condition modelling power-offs. The latter condition seemed to be effective, as it explicitly uses the ratio of idle servers to the total number of servers.

The next step is to find the combination of parameter values that minimises the objective function. Since they are continuous and relatively numerous, the search space is very large. Thus to explore it and find an optimal solution a meta-heuristic algorithm, namely Particle Swarm Optimisation (PSO), is used.

\subsection{Particle swarm optimisation}

As mentioned in the previous section, the goal of the particle swarm optimisation (PSO) is not to directly find an optimal solution to schedule the different jobs but rather to find an optimal set of parameters that could improve the decision processes of the greedy algorithm.

PSO is a nature-inspired algorithm that imitates the behaviour of animal flocks. A Swarm is a group of particles which are mobile entities characterised by their position, velocity and cost. A swarm converges
Table 2

Overall parameters of the greedy algorithm.

\begin{tabular}{lllll}
\hline \multicolumn{2}{l}{ Overall reconfiguration parameters } & & & \\
\hline$w_{n}$ & $w_{\alpha}$ & $s_{\text {reconfig }}$ & $w_{D}$ & bias \\
\hline $\begin{array}{l}\text { Servers count } \\
\text { weight }\end{array}$ & $\begin{array}{l}\text { Speedup } \\
\text { factor } \alpha\end{array}$ & $\begin{array}{l}\text { Scaling } \\
\text { factor }\end{array}$ & $\begin{array}{l}\text { Data } \\
\text { weight }\end{array}$ & $\begin{array}{l}\text { Bias } \\
\text { term }\end{array}$ \\
& weight & & & \\
\hline Power off parameters & $s_{\text {off }}$ & $t_{1_{\text {off }}}$ & $t_{2_{\text {off }}}$ & $p_{t_{t_{\text {off }}}}$ \\
\hline$w_{\text {off }}$ & Scaling & Duration & Duration & $t_{1_{\text {off }}}$ \\
\hline $\begin{array}{l}\text { Available } \\
\text { servers count }\end{array}$ & factor & 1 & 2 & probability \\
weight & & & &
\end{tabular}

towards an ideal location as its individuals move with respect to the group best-known position and their respective best-known position. This technique enables for an exploration of the search space while converging towards an optimal solution [17]. The used algorithm is similar to the one described in [18]. It uses the reflect method to deal with boundary escape from [19]. The used PSO algorithm is outlined in Algorithm 2.

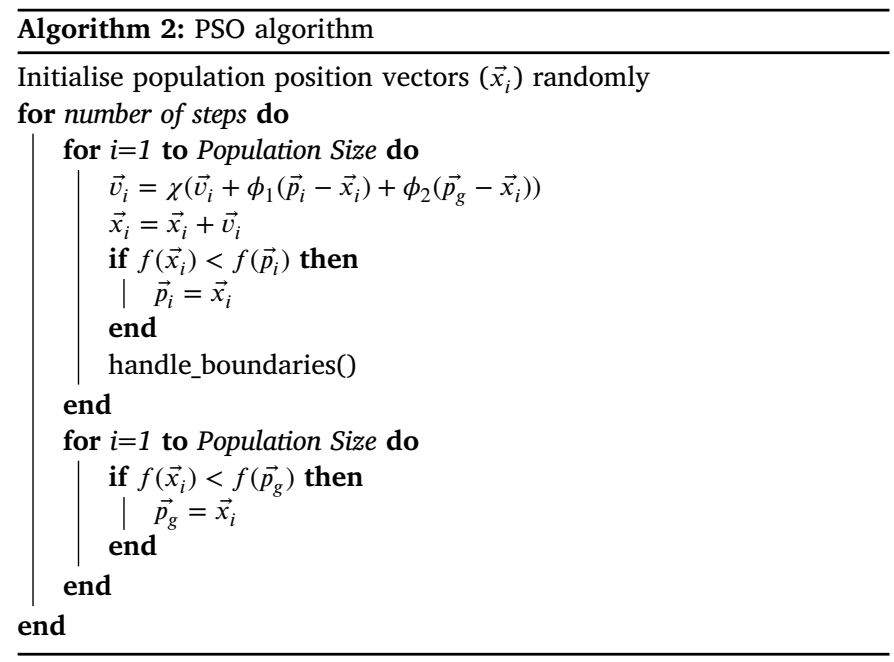

In our experiments, the set of parameters is either represented as an 8-dimensional vector or a 9-dimensional vector. $\vec{x}_{i}$ depicts the position of the $i$ th particle. Each particle has a velocity vector $\vec{v}_{i}$, used to update the vector $\vec{x}_{i}$, at every step. $\vec{v}_{i}$ is calculated using:

$\vec{v}_{i}=\chi\left(\vec{v}_{i}+\phi_{1}\left(\vec{p}_{i}-\vec{x}_{i}\right)+\phi_{2}\left(\vec{p}_{g}-\vec{x}_{i}\right)\right)$

where $\chi$ is a constant influencing the magnitude of update. It was found that a value of 0.1 works well compared to higher values that tend to push particles out of bounds too quickly. $\vec{p}_{i}$ is the particle best-known position, $\vec{p}_{g}$ is the global best position. $\phi_{1}$ and $\phi_{2}$ are random numbers between 0 and 2 generated for each particle at each time step. The cost function $f$ for a particular configuration is a function mapping a position vector to a real number. As for boundary escape, if a parameter $j$ of $\vec{x}_{i}$ goes below or above the $x_{j_{\text {_min }} / \max }$ thresholds then it is handled as follows:

$x_{i j}=x_{\text {jmax }}-\left(x_{i j}-x_{\text {max }}\right)$ if $x_{i j}>x_{\text {max }}$

$x_{i j}=x_{j \min }+\left(x_{j \min }-x_{i j}\right)$ if $x_{i j}<x_{j \min }$

\section{Experimental setup}

\subsection{Simulation environment}

As there are no known simulators - to our knowledge - supporting the scheduling of malleable jobs, a simulation environment was developed using python to test the performance of the designed 


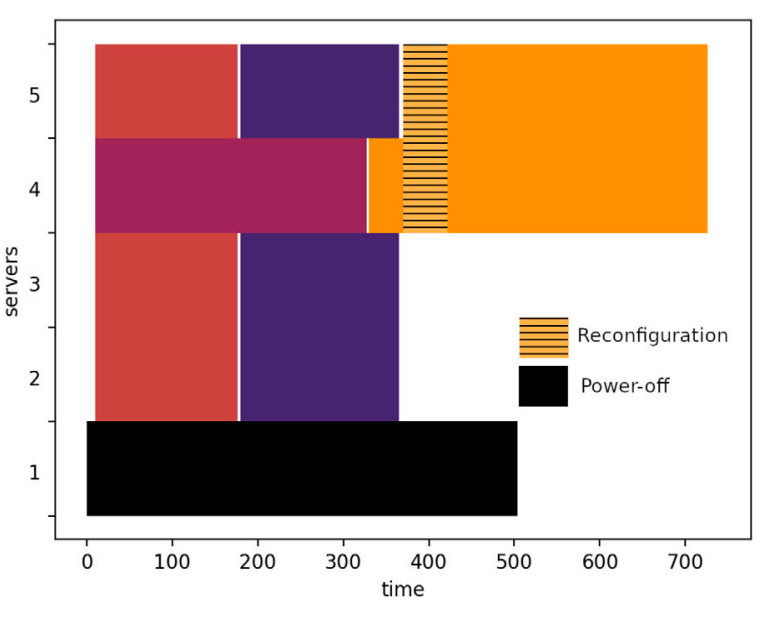

Fig. 2. Visual output from the simulator.

Table 3

Parameters used for the generation of jobs.

\begin{tabular}{ll}
\hline Parameter & Used value \\
\hline Server count & 10 \\
Job count & 50 \\
Dynamism & 500 \\
Mass & 1700 \\
Disparity & 3.8 \\
Speed-up factor $(\alpha)$ & $U(0.5,1)$ \\
Data & $U(10,500)$ \\
\hline
\end{tabular}

scheduling strategy. At the time of writing this paper, it only provided reconfiguration of jobs from a smaller to a larger number of servers as it was thought to be more relevant in decreasing the mean stretch time. An example of the visualisation output of the simulator can be seen in Fig. 2.

\subsection{Jobs generation}

To simulate a realistic environment, Google data centres-like jobs were generated similar to [20]. The only difference lies in the way a job is defined. In [20] jobs are sets of small sequential tasks schedulable in a particular order. In our approach, a job is a single parallelisable task. A uniform random distribution is used to generate parameters such as the speed-up factors, the data and the required number of servers. All the undertaken experiments generated 50 jobs to be scheduled on ten servers. A summary of the job generation parameters is shown in Table 3 where the dynamism is the average duration between two consecutive jobs and the disparity is the ratio of the average makespan to the median makespan. The values for the mass and disparity correspond to the average values obtained in [20].

\subsection{Particle swarm search of parameters}

Since the number of parameters to tune is based on which condition is used for reconfiguration, three different swarms of 30 particles each were trained. Swarm1 (training), Swarm2 (training) and Swarm3 (training) use respectively condition1, condition2 and condition3. Each particle of each trained swarm was assigned set of valid parameters following the laws described in [20] and the values in Table 3. At each epoch $k$ a set $G_{k}$ is generated, where $G_{k}=\left\{S_{1}, S_{2}, \ldots, S_{50}\right\}$ and $S_{i}$ is a set of 50 different jobs. Every particle had to schedule $G_{k}$ independently. This partitioning of the set of jobs $G_{k}$ into smaller subsets $S_{i}$ is used to prevent the parameters within particles from overfitting a particular set of jobs. It also ensures that the job generator is updated with newer seeds guaranteeing diversity among the jobs. The scheduling of one set

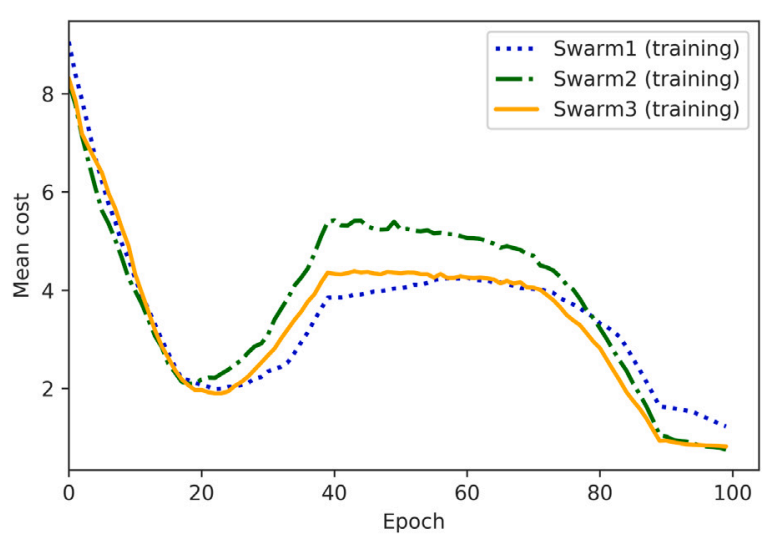

Fig. 3. Mean cost of all the particles against the number of epochs during training.

Table 4

Configuration of parameters found by PSO of each of the trained swarms.

\begin{tabular}{llll}
\hline \multicolumn{1}{c}{ Swarm1 } & Swarm2 & Swarm3 \\
\hline \multicolumn{2}{l}{ Reconfiguration parameters } & & \\
\hline$w_{n}$ & 0.175 & 0.348 & 0.529 \\
$w_{\alpha}$ & 0.742 & 0.833 & 0.645 \\
$s_{\text {reconfig }}$ & 0.331 & 0.579 & $N / A$ \\
$w_{D}$ & $N / A$ & 0.730 & 0.289 \\
bias & $N / A$ & $N / A$ & -0.106 \\
\hline Power off parameters & & & \\
\hline$w_{\text {off }}$ & 0.455 & 0.516 & 0.494 \\
$s_{\text {off }}$ & 0.760 & 0.814 & 0.813 \\
$t_{1 \text { off }}$ & 899 & 528 & 632 \\
$t_{2 \text { off }}$ & 1405 & 2962 & 1233 \\
$p_{t_{\text {loff }}}$ & 0.717 & 0.959 & 0.615 \\
\hline
\end{tabular}

$S_{i}$ is further referred to as an experiment. The cost function is evaluated for each particle, by taking the average cost of the $50 S_{i}$ within $G_{k}$. Also, every set of generated jobs $G_{k}$ at epoch $k$ is different from the one generated at epoch $k+1$. The total number of used epochs is 100 . This value was chosen as it was noticed that all particles had converged towards the same set of parameters and there was little change from one epoch to the next.

The mean cost of all the particles as a function of the number of epochs for each trained swarm is depicted in Fig. 3.

The graph shows that for each of the swarms, the parameter search went through three different phases. From epoch one to about 20, there is a clear improvement in minimising the cost function. Then from epoch 20 to about 70, particles still have quite different sets of parameters. Yet, these parameters tend to get closer to one another. At this point, the swarm is exploring the neighbourhood of the minimum it would later settle in, which justifies the flattening of the cost values from about epoch 40 to 70 . From epoch 70 to 100, the mean cost slowly decreases until convergence, and the swarm finds a minimum optimising the cost function. So the three trained swarm converged at around the same range of epochs. Moreover, those similarities in the cost pattern can be explained by the fact that the training took place under the same seed and that the job generator had not been modified, which resulted in having the same job distribution for each of the swarms. This shows that the performance of the scheduler is highly dependent on the set of jobs to be scheduled. The found parameters are shown in Table 4.

The implication of the found results will be discussed in more details in the next section. Nevertheless, it is already worth noting that even though every particle's parameters are initialised randomly, every trained swarm learned two different shutdown time durations a short one for $t_{1_{\text {off }}}$ and a long one for $t_{2_{o f f}}$, and whenever a poweroff takes place at least $60 \%$ of the times, it lasts for a shorter amount 
of time. The difference in values for the shutdown times found by Swarm 2 is quite large. In fact $t_{1 \text { off }}$ is close to the minimum required duration for energy-saving whereas $t_{2 \text { of } f}$ is more than twice as large as the values found by Swarm 1 and Swarm3. However the probability for choosing $t_{1 \text { off }}$ over $t_{2 \text { off }}$ in Swarm 2 is about $96 \%$, meaning that longer shutdown times will be rare. No indication was given in the initial values of the particles that one time should be longer than the other.

\subsection{Comparison setups}

To evaluate the performance of the scheduler, ten different experimental setups are introduced and run under identical conditions. In each of these setups, jobs are generated such there is only one big set of sets of jobs $G_{1}$ that is composed of 100 subsets $S_{i}$, and where each $S_{i}$ contains 50 different jobs to be scheduled on ten identical servers. The main distinction between the first four setups and the last six lies within the decision-making process, which is based on the parameters defined in Table 2. In the first four setups, the reconfigurations and power-offs are performed whenever possible without any involvement of the parameters. Whereas the last six setups rely on those parameters to make a decision.

- FIFO (FIFO): The base case. It is only the first step of the greedy algorithm, a naive FIFO scheduler where the jobs are scheduled according to their submission time.

- FIFO \& Reconfigurations (FIFO-Rcfg): A FIFO scheduler that always reconfigures jobs whenever servers are available.

- FIFO \& Power off (FIFO-Poff): A FIFO scheduler that always shuts down idle servers - when possible - for a constant downtime of $900 \mathrm{~s}$.

- FIFO \& Reconfigurations \& Power off (FIFO-Rcfg-Poff): A FIFO scheduler combining reconfigurations and power-offs in the same fashion as in FIFO-Poff and FIFO-Rcfg.

- Random parameters (Rand-Param1): A FIFO scheduler that uses condition1 to reconfigure jobs and shuts down idle servers based on randomly generated parameters.

- Random parameters (Rand-Param2): A FIFO scheduler that uses condition2 to reconfigure jobs and shuts down idle servers based on randomly generated parameters.

- Random parameters (Rand-Param3): A FIFO scheduler that uses condition 3 to reconfigure jobs and shuts down idle servers based on randomly generated parameters.

- With Swarm1 parameters (Swarm1): A FIFO scheduler that uses condition 1 and that is capable of reconfiguring jobs and shutting down servers based on the PSO parameters found by Swarm1 (training).

- With Swarm2 parameters (Swarm2): A FIFO scheduler that uses condition2 and that is capable of reconfiguring jobs and shutting down servers based on the PSO parameters found by Swarm2 (training).

- With Swarm3 parameters (Swarm3): A FIFO scheduler that uses condition 3 and that is capable of reconfiguring jobs and shutting down servers based on the PSO parameters found by Swarm3 (training).

\section{Results}

In this section, the method used for ranking the different setups is outlined. The results of the experiments are analysed by first looking at the number of power-offs and reconfigurations performed by each setup. Then, the individual components of the cost are investigated. Following, a statistical analysis of the overall costs is performed. Finally a resilience test on the best performing setup along with a scale-up test are presented.

\subsection{Ranking methodology}

To draw conclusions from the results, a ranking between each of the setups is established. In this section, the statistical method for the ranking is described. The first step of the approach is to rank each setup for each scheduled set of jobs. A rank of 1 denotes the best performing setup (lowest mean cost) and a rank of 10 the worst performing one. As one hundred sets $S_{i}$ of jobs were used for the experiment, one hundred rankings are obtained. Then, the average ranking of each setup is calculated. To evaluate whether those average ranks are significantly different from the mean rank (5.5), the Friedman $\chi_{F}^{2}$ and its corresponding $p$-value are used. Thus for a confidence level of $95 \%$ corresponding to an $\alpha$ value of 0.05 , it is meaningful to perform post-hoc tests as described in [21] if the $p$-value is less than 0.05 . The post-hoc tests make it possible to identify significant pairwise differences among the different setups. This is done by first finding the standard error (SE) between two setups using:

$S E=\sqrt{\frac{k(k+1)}{6 N}}=\sqrt{\frac{10 \times 11}{6 \times 100}}=0.265$

where $k$ is the setup count and $N$ the number of experiments. Using the average ranks and the standard error the $z$-values are computed using:

$z=\frac{R_{i}-R_{j}}{S E}$

where $R_{i}$ and $R_{j}$ are the average ranks of two different setups. From the $z$-values, the corresponding probabilities ( $p$-values) are found. The null hypothesis is that the difference in ranks is due to chance. However, if a $p$-value is below $\alpha=0.05$, then the null hypothesis can be rejected with level of confidence of $95 \%$.

\subsection{Reconfigurations and power-offs results}

Table 5 shows the average number of reconfigurations and the average number of power-offs per setup. The most striking result is that the average number of reconfigurations performed by the setup Swarm1 is zero. This implies that the parameters do not allow for any reconfigurations to take place for the generated jobs. The parameters from Swarm2 allow for very few reconfigurations to take place. This is also an unexpected result as one would expect that reconfigurations could improve the average stretch time. This result seems to highlight the difficulty of deciding when to perform reconfigurations and that if they are not carefully undertaken the performance might not improve. The average number of reconfigurations made by the three setups RandParam show that the improved cost reached by the Swarm setups is due to the optimisation process and not just the choice of the equation describing the decision process. The average numbers of power-offs for the three Swarm setups are of the same order of magnitude which can be explained by the fact that the equations and parameters governing the power-off decision process for those three setups are the same. The different Swarm based set-ups allow for a relatively large number of power-offs. It may seem that this could cause power spikes and have a negative impact on the energy saved. However modern hardware can slightly shift the time of powering different servers on and off so that they do not all take place at the same time and therefore avoiding power spikes.

\subsection{Ranking by the mean stretch time}

Fig. 4 shows the distributions of the mean stretch time metric obtained from conducting 100 experiments for each of the different setups. Although it cannot be seen on the graphs, the setup FIFO-RfcgPoff has a maximum mean stretch time (around 80) that is much higher than in any of the other setups. The distribution from FIFO-Poff also suggests that mean stretch time was higher than for the other setups in some instances. The distributions of the mean stretch times of the 
Table 5

Average number of reconfigurations and power-offs of each setup.

\begin{tabular}{lll}
\hline & $\mu_{\text {reconfigs }}$ & $\mu_{P_{o f f}}$ \\
\hline FIFO & 0.00 & 0.00 \\
FIFO-Rcfg & 3.07 & 0.00 \\
FIFO-Poff & 0.00 & 228.38 \\
FIFO-Rcfg-Poff & 13.30 & 458.51 \\
Rand-Param1 & 3.69 & 82.92 \\
Rand-Param2 & 0.00 & 56.33 \\
Rand-Param3 & 0.04 & 0.00 \\
Swarm1 & 0.00 & 139.11 \\
Swarm2 & 0.01 & 216.50 \\
Swarm3 & 5.04 & 160.21 \\
\hline
\end{tabular}

Table 6

Setups ranking by the mean stretch time.

\begin{tabular}{ll}
\hline Rank & Setup \\
\hline 1 & Swarm2 \\
2 & Swarm3 and Swarm1 \\
3 & FIFO and Rand-Param1 and Rand-Param3 \\
4 & FIFO-Rcfg and Rand-Param2 \\
5 & FIFO-Rcfg-Poff and FIFO-Poff \\
\hline
\end{tabular}

Table 7

Setups ranking by the mean normalised power.

\begin{tabular}{ll}
\hline Rank & Setup \\
\hline 1 & FIFO-Poff and Swarm1 \\
2 & Swarm2 and Swarm3 \\
3 & FIFO-Rcfg-Poff and Rand-Param2 \\
4 & Rand-Param1 \\
5 & FIFO and Rand-Param3 \\
6 & FIFO-Rcfg \\
\hline
\end{tabular}

setups using the decision equations seem to have similar shapes for random parameters and PSO-obtained parameters. Another setup pair with similar distributions corresponds to FIFO and FIFO-Rcfg. This is not surprising as the logic between these two setups is quite similar. Using the statistical methodology previously described, the Friedman $\chi_{F}^{2}$ is found to be equal to 442.05 which corresponds to a $p$-value of $1.43 \times 10^{-89}$ which signifies that it is meaningful to perform post-hoc tests to identify significant pairwise differences. The ranking obtained with a confidence level of $95 \%$ is shown in Table 6. Clearly, the setups using the PSO-obtained parameters ranked better than the other setups. The setups with the worst performances are FIFO-Poff and FIFO-RcfgPoff. This result can be explained by the fact that if the power-offs are not scheduled properly then the mean stretch time increases as the servers may be off at times when there is high demand.

\subsection{Ranking by the mean normalised power consumption}

Similarly to the previous section, Fig. 5 shows the different distributions of the average normalised power metric of each setup. The range of values seems to vary over a smaller range from one setup to another compared to the stretch times shown in the previous section. This is expected as power is a measure of the rate of energy transformation, and there is a narrower range of allowed values (with respect to the states of the servers) at a given time. Unsurprisingly the setups that do not allow performing power-offs have a higher minimum average normalised power. The Friedman $\chi_{F}^{2}$ is found to be equal to 831.72 which corresponds to a $p$-value of $3.15 \times 10^{-173}$ which signifies that it is meaningful to perform post-hoc tests to identify significant pairwise differences. The ranking obtained with a confidence level of $95 \%$ is shown in Table 7. As expected, the FIFO-Poff setup that allows performing power-offs whenever possible ranks best on this metric. The setups that did not allow for any power-offs rank last. Again the three Swarm setups rank among the best ones.
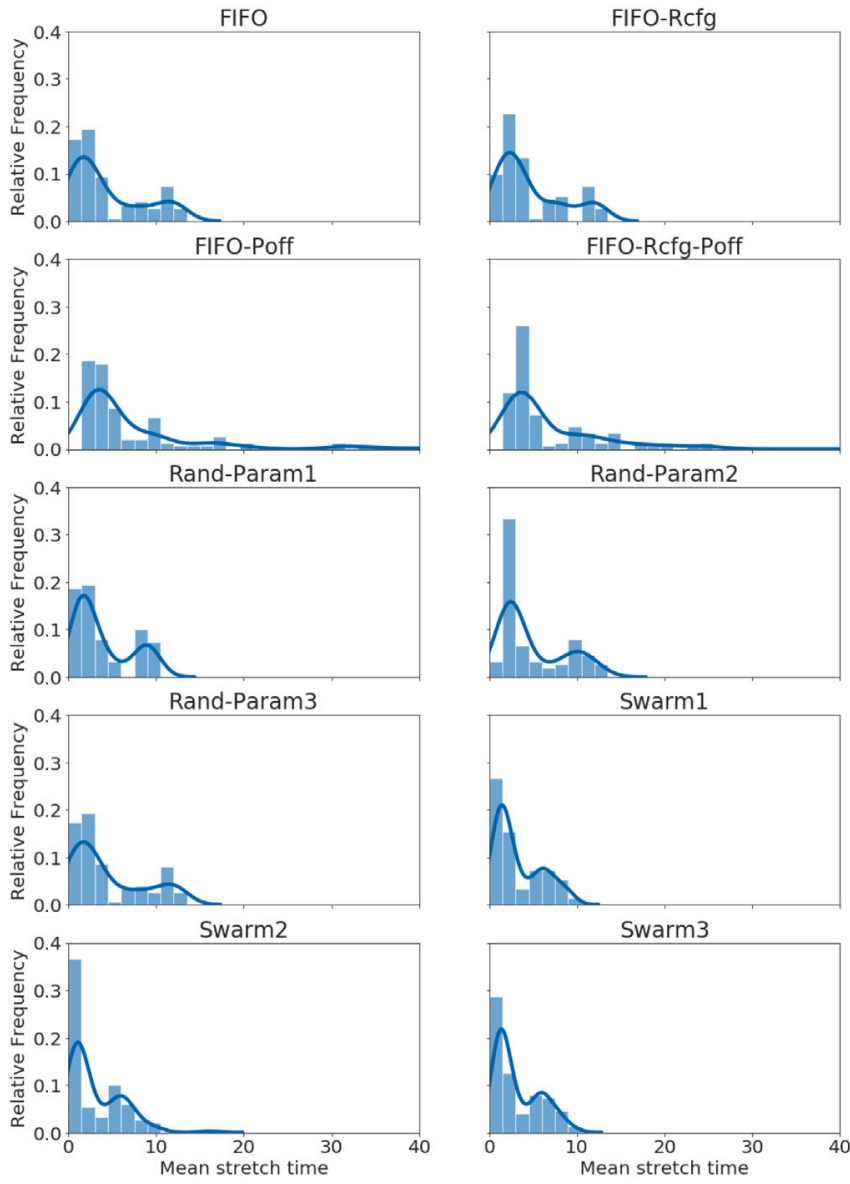

Swarm 1

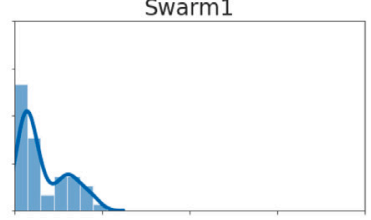

Swarm3

Fig. 4. Histograms of the mean stretch time of each setup.

\subsection{Ranking by the overall cost}

In this section the obtained cost values are compared to identify which setup may lead to a better overall performance. As a reminder, the cost function is defined as the product of the mean stretch time and the mean normalised power.

Fig. 6 shows the distribution of the overall cost values obtained from conducting 100 experiments in each setup. It seems that the cost values of Swarm1, Swarm2 and Swarm3 are more skewed towards a lower cost than in the other setups.

Fig. 7 shows pairwise comparisons of the number of times a particular setup obtained a lower cost value than an another setup. Clearly the three Swarm setups perform better than all the other setups on the majority of the experiments.

The average ranking over the 100 experiments can be seen in Table 8. It can be noted that no setup has an average rank that is close to 1 , meaning that there is probably no setup that outranks all the others on most occasions.

The Friedman $\chi_{F}^{2}$ is in this case equal to 448.09 which corresponds to a $p$-value of $7.33 \times 10^{-91}$. Thus for a confidence level of $95 \%$ corresponding to an $\alpha$ value of 0.05 , it is meaningful to perform posthoc tests to identify significant pairwise differences among the different setups. The weak ranking among the six setups is shown in Table 9.

The worst performing setup is FIFO-Rcfg-Poff. It implies that reconfigurations and power-offs require a careful decision-making process to ensure an increase in performance, and that it is not beneficial to perform them whenever it is possible. The three Swarm setups are all ranked first, which can be explained by the exploration of the parameter space that allowed the algorithms to 'learn' to react to the job 

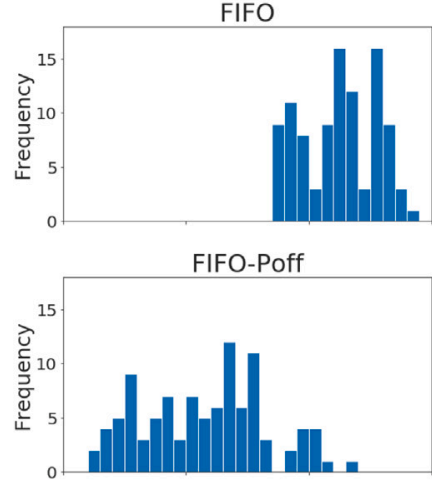

Rand-Param

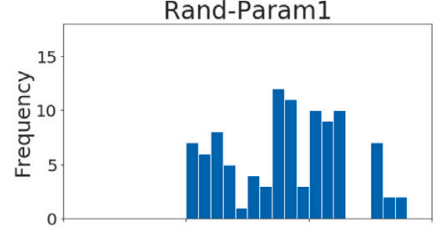

Rand-Param3

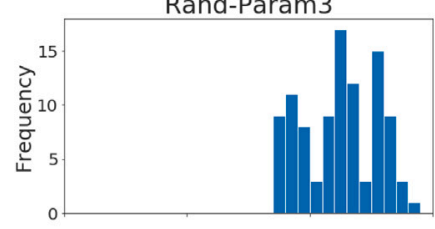

Swarm2

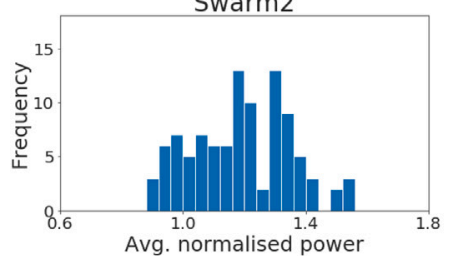

Fig. 5. Histograms of the mean normalised power of each setup.

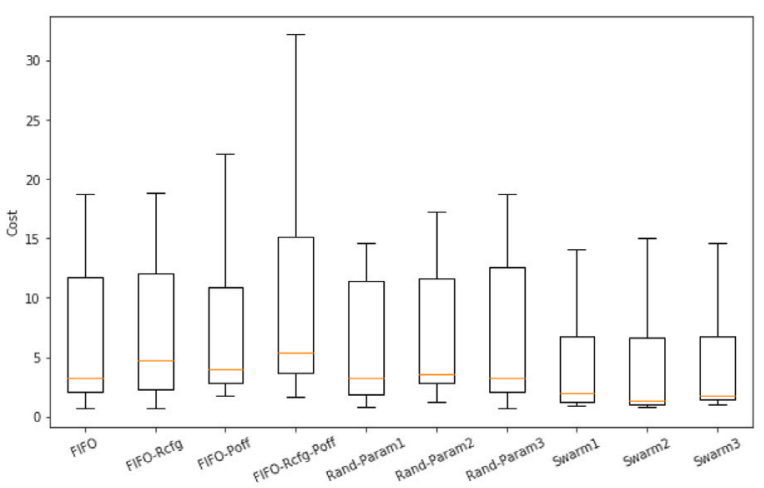

Fig. 6. Boxplots of the cost of each setup.

generator's distribution. Interestingly, there is no statistical evidence that FIFO performs better than Rand-params1 or Rand-params 3 as they are all ranked second. To evaluate the significance of this ranking, a resilience test is described in the next section.

\subsection{Resilience of PSO parameters}

To test the ability of the three Swarm setups to respond to changes in the job generator's distribution, the setups were re-run under identical conditions to the ones in the previous section. The parameters of the job

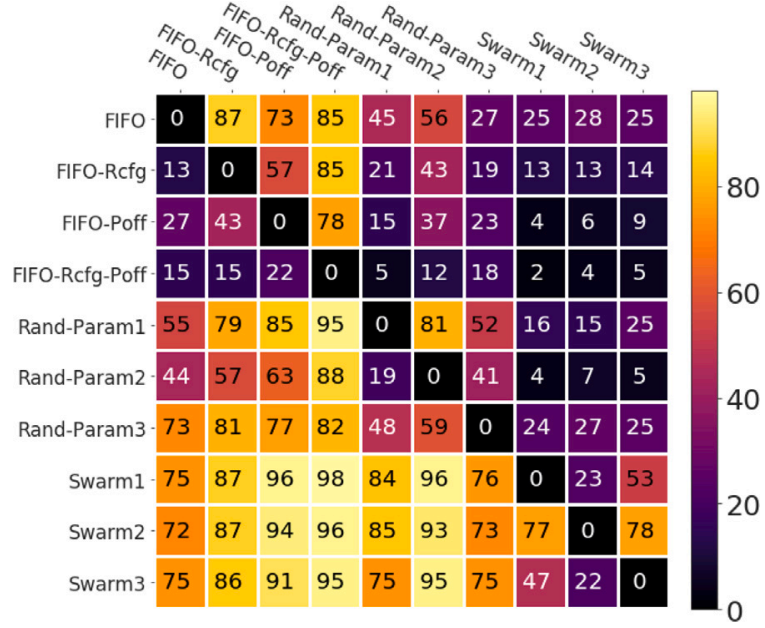

Fig. 7. Heatmap of the performance pairwise comparisons between setups on the cost criterion.

Table 8

Average ranking of each setup over the 100 experiments.

\begin{tabular}{ll}
\hline Setup & Average ranking \\
\hline FIFO & 5.49 \\
FIFO-Rcfg & 7.22 \\
FIFO-Poff & 7.58 \\
FIFO-Rcfg-Poff & 9.02 \\
Rand-Param1 & 4.97 \\
Rand-Param2 & 6.72 \\
Rand-Param3 & 5.07 \\
Swarm1 & 3.12 \\
Swarm2 & 2.45 \\
Swarm3 & 3.39 \\
\hline
\end{tabular}

Table 9

Setups ranking by the overall cost.

\begin{tabular}{ll}
\hline Rank & Setup \\
\hline 1 & Swarm1 and Swarm2 and Swarm3 \\
2 & FIFO and Rand-Param1 and Rand-Param3 \\
3 & Rand-Param2 and FIFO-Rcfg and FIFO-Poff \\
4 & FIFO-Rcfg-Poff \\
\hline
\end{tabular}

Table 10

Jobs generator parameters used in the resilience test.

\begin{tabular}{lll}
\hline Parameter & Previous value & New value \\
\hline Server count & 10 & 10 \\
Job count & 50 & 50 \\
Dynamism & 500 & 300 \\
Mass & 1700 & 1900 \\
Disparity & 3.8 & 4.5 \\
Speed-up factor $\alpha$ & $U(0.5,1)$ & $U(0.5,1)$ \\
Data & $U(10,500)$ & $U(10,800)$ \\
\hline
\end{tabular}

generator were slightly adjusted to simulate an environment where the frequency, the mass and the data of the jobs increased. Thus simulating an increase in the demand. The change of values can be found in Table 10.

Table 11 shows that this time the Swarm1, Swarm2, Rand-Param2 and Rand-Param 3 allowed performing reconfigurations. This shows that the decision process of reconfigurations is sensitive to the distribution of the jobs and that a slight change in the job's parameters results in a different scheduling strategy.

Fig. 8 displays the different distributions of the costs. It can be seen that the setup FIFO-Poff that allows for power-offs to be performed whenever possible seems to have an overall cost distribution skewed towards lower values. This can be explained by the fact that this setup 
Table 11

Average number of reconfigurations and power-offs of each setup in the resilience test.

\begin{tabular}{lll}
\hline & $\mu_{\text {reconfigs }}$ & $\mu_{P_{o f f}}$ \\
\hline FIFO & 0.00 & 0.00 \\
FIFO-Rcfg & 4.34 & 0.00 \\
FIFO-Poff & 0.00 & 297.33 \\
FIFO-Rcfg-Poff & 5.77 & 189.25 \\
Rand-Param1 & 2.92 & 20.27 \\
Rand-Param2 & 3.63 & 11.19 \\
Rand-Param3 & 2.50 & 0.00 \\
Swarm1 & 7.53 & 87.32 \\
Swarm2 & 1.23 & 184.27 \\
Swarm3 & 4.85 & 128.89 \\
\hline
\end{tabular}

Table 12

Setups ranking during the resilience test.

\begin{tabular}{ll}
\hline Rank & Setup \\
\hline 1 & Swarm2 \\
2 & FIFO \\
3 & Swarm3 and FIFO-Rcfg and Swarm1 and FIFO-Poff \\
4 & FIFO-Rcfg-Poff and Rand-Param1 and Rand-Param3 \\
5 & Rand-Param2
\end{tabular}

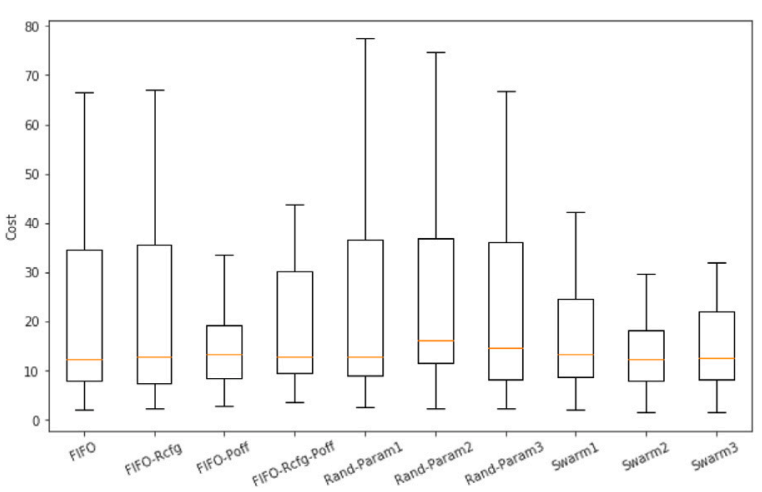

Fig. 8. Cost of each used setup in the resilience test.

is the one that performs the highest number of power-offs per set of jobs thus entailing a lower average power consumption. This does not necessarily imply that it is a beneficial setup as the mean stretch time can be very large. The Friedman Chi-square value $\chi_{F}^{2}=165.67$ and its corresponding $p$-value $\left(4.93 \times 10^{-31}\right)$ suggest that post-hoc testing can be meaningful. This time the ranking cannot be as clearly established as in the previous section and the weak ranking displayed in Table 12 was produced using a confidence level of $90 \%$. This time again Swarm2 ranks first. However, the other two setups Swarm1 and Swarm3 did not perform as well as they rank third. It is worth mentioning that the simple FIFO scheduler that performs neither reconfigurations nor power-offs ranks second. Unsurprisingly using random parameters for the decision processes leads to a poor overall performance.

\subsection{Scale up test}

The scale-up test aims at simulating a more realistic environment by increasing the number of servers from 10 to 100 while keeping the average number of submitted jobs per server per unit time the same as before. Similar to the resilience test, the goal is to test how the different setups would behave in an environment that is different from the one in which the optimisation process took place. The change in the parameters made during the scale-up test can be found in Table 13.

As shown in Table 14, the average number of reconfigurations and power-offs performed by the three Swarm setups during the scale-up test are similar to the ones of the initial experiment. This shows if the jobs distribution is kept the same on a larger scale, the three
Table 13

Scale-up parameters.

\begin{tabular}{lll}
\hline Parameter & Previous value & New value \\
\hline Server count & 10 & 100 \\
Job count & 50 & 500 \\
Dynamism & 500 & 50 \\
Mass & 1700 & 1700 \\
Disparity & 3.8 & 3.8 \\
Speed-up factor $\alpha$ & $U(0.5,1)$ & $U(0.5,1)$ \\
Data & $U(10,500)$ & $U(10,500)$ \\
\hline
\end{tabular}

Table 14

Average number of reconfigurations and power-offs during the scale-up test.

\begin{tabular}{lll}
\hline & $\mu_{\text {reconfigs }}$ & $\mu_{P_{\text {off }}}$ \\
\hline FIFO & 0.00 & 0.00 \\
FIFO-Rcfg & 76.35 & 0.00 \\
FIFO-Poff & 0.00 & 266.17 \\
FIFO-Rcfg-Poff & 75.40 & 233.41 \\
Rand-Param1 & 0.00 & 180.01 \\
Rand-Param2 & 30.74 & 0.00 \\
Rand-Param3 & 0.00 & 0.00 \\
Swarm1 & 0.00 & 150.76 \\
Swarm2 & 0.11 & 192.80 \\
Swarm3 & 12.63 & 170.75 \\
\hline
\end{tabular}

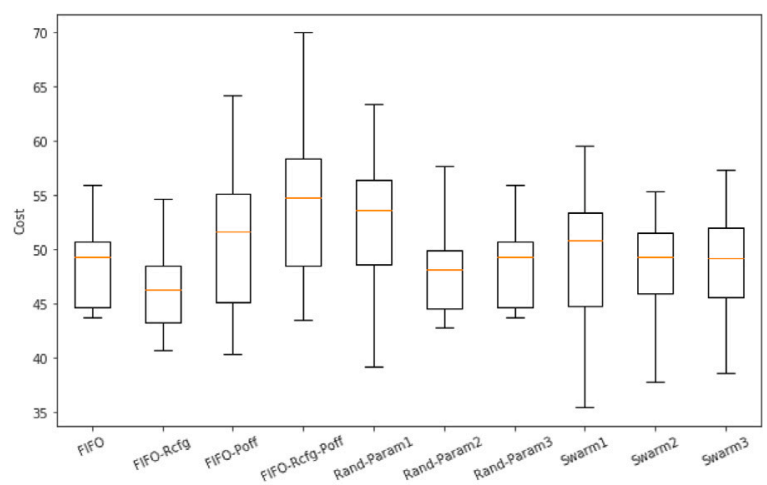

Fig. 9. Cost of each used setup in the scale up test.

Swarm setups behave consistently. However, this is not the case for the setups using random parameters, as Rand-Param2 that did not initially allow for reconfigurations, now performs a large average number of reconfigurations. Table 14 shows the cost distribution of the different setups. As shown in Fig. 9 the three Swarm setups have a whisker in the lower costs that is longer than in Fig. 6 and Fig. 8. This implies that the lower quartiles spreads over a larger range of costs than in the previous two tests. There is some overlap of the different distributions and further analysis needs to be conducted to establish a ranking. The Friedman Chi-square value $\chi_{F}^{2}=358.68$ and its corresponding $p$-value $\left(8.80 \times 10^{-72}\right)$ suggest that post-hoc testing can be meaningful. The weak ranking is shown in Table 15. The result is rather unexpected as the setup FIFO-Rcfg that allowed for reconfigurations to take place whenever possible ranks first. A large number of servers provides a larger number of possible ways to schedule a given job. A reconfiguration is less likely to have a large effect on the fractional number of servers being used if the number of servers is large. This result can also be explained by the fact that the setup FIFO-Rcfg performed really well under the mean stretch time metric, it did however perform very poorly on the average power metric. The different three Swarm setups ranked equally well at rank 4 despite a behaviour that is consistent with the initial test. When scaling up there does not seem to be a significant advantage in using one of the decision equation over any of the other ones. The setup that allows for reconfigurations and power-offs to take place whenever possible ranks last similar to all the previous tests. 
Table 15

Setups ranking by overall cost in the scale-up test.

\begin{tabular}{ll}
\hline Rank & Setup \\
\hline 1 & FIFO-Rcfg \\
2 & Rand-Param2 \\
3 & Rand-Param3 and FIFO \\
4 & Swarm1 and Swarm2 and Swarm3 \\
5 & FIFO-Poff \\
6 & Rand-Param1 \\
7 & FIFO-Rcfg-Poff \\
\hline
\end{tabular}

\subsection{Discussion of results and limitations}

The results show that using a decision process for reconfigurations and power-offs can lead to a low overall cost. This decision process is based on the scheduler state and made use of parameters that were tuned using PSO. However, this performance was found to be sensitive to changes in the way jobs are submitted and the total number of servers in the HPC cluster. The difficulty with taking energy consumption as a metric is that minimising it by turning off servers can have a negative impact on the jobs waiting time in the queue. Therefore a setup that ranks well on one of the metrics does not necessarily rank well on the other. It would be interesting during the PSO training to have variable weights over the metrics within the objective function. It would prevent that one metric gets favoured over the other. This was probably the case with the setups that did not allow for reconfigurations to take place. One of the limitations is that the PSO training can take a long time, especially if the number of servers and jobs are large. Our implementation could be improved by using multi-processing and generating jobs in advance so that they do not have to be re-generated every time a swarm has to be trained. This would increase the speed of experimentation with the proposed equations for the decision process. In this investigation, the job generator simulates a realistic distribution for the frequency at which jobs are submitted and their mass. But, both the speed-up factors and the data amount are drawn from uniform random distributions. Using a more realistic distribution for those parameters might improve the ability of the PSO to find patterns in the jobs. Finally, another aspect that is not taken into consideration in this investigation is that turning servers on and off frequently may affect their lifetime. This is particularly important when considering the environmental aspect, as energy is not only required when the servers are used but also when they are manufactured and disposed of.

\section{Conclusions \& perspectives}

This paper presented a solution that aims at improving the performance of HPC schedulers by lowering both the mean stretch time and the average power consumption by relying on malleable job reconfigurations and server power-offs. As current simulators do not support the scheduling of malleable jobs, we implemented our own simulator. The job generator's distribution was modelled based on real data. The proposed algorithm consisted of three steps: FIFO scheduling, reconfiguration attempts and powering off of the remaining idle servers. Various parameters and conditions were used to decide whether reconfigurations and power-offs should be performed. It was assumed that the scheduler has no knowledge about any future states and used only local context knowledge to make decisions.

Three different equations were used to decide whether a reconfiguration should take place and the parameters of those equations were tuned using PSO. It was found that the three different setups using the PSO-obtained parameters performed equally well and better than the other setups as long as the distribution of jobs is similar to the one it was trained on. If the frequency at which jobs are submitted as well as their average masses are increased, one of the setups using PSO-obtained parameters ranks first with a level of confidence of $90 \%$, but the other two did not perform so well. If the number of servers is increased to a more realistic number (100) then it was found that the setup that only used the FIFO scheduling as well as reconfigurations whenever possible performed best. However, this performance was at the expense of the average power metric.

We believe that the malleable job paradigm should be investigated further by, for example, allowing jobs to be assigned to a lower number of servers if the queue becomes too long. This would require keeping some statistics about the average length of the queue at a given time. Being able to accurately predict the remaining mass of a job at a given time would facilitate the evaluation of whether a reconfiguration could be beneficial. Probably one of the biggest challenges remains to find a suitable encoding for malleable tasks so that other meta-heuristic algorithms could be used to find more optimal solutions.

\section{CRediT authorship contribution statement}

Briag Dupont: Conceptualization, Methodology, Software, Formal analysis, Investigation, Data curation, Writing - original draft, Writing - review \& editing, Visualization. Nesryne Mejri: Conceptualization, Methodology, Software, Validation, Investigation, Data Curation, Writing - original draft, Writing - review \& editing, Visualization. Georges Da Costa: Conceptualization, Supervision, Project administration, Funding acquisition.

\section{Declaration of competing interest}

No author associated with this paper has disclosed any potential or pertinent conflicts which may be perceived to have impending conflict with this work. For full disclosure statements refer to https://doi.org/ 10.1016/j.suscom.2020.100447.

\section{Acknowledgements}

The work presented in this paper has been funded by the ANR in the context of the project ENERGUMEN, ANR-18-CE25-0008.

We would like to thank Prof. Pascal Bouvry and Dr. Grégoire Danoy for their guidance throughout the project.

We would also like to thank Luc Sinet for his invaluable advice on software development and Lucas Romao for his support.

\section{References}

[1] H. Ferreboeuf, Lean ICT: Towards Digital Sobriety, Tech. rep., The Shift Project, 2018.

[2] F. Pinel, J. Pecero, S. Khan, P. Bouvry, Energy-efficient scheduling on milliclusters with performance constraints, in: Proceedings - 2011 IEEE/ACM International Conference on Green Computing and Communications, GreenCom 2011, 2011, pp. 44-49, http://dx.doi.org/10.1109/GreenCom.2011.16.

[3] V. Villebonnet, G. Da Costa, L. Lefevre, J.-M. Pierson, P. Stolf, Energy Aware Dynamic Provisioning for Heterogeneous Data Centers, 2016, pp. 206-213, http://dx.doi.org/10.1109/SBAC-PAD.2016.34.

[4] J. Shalf, S. Dosanjh, J. Morrison, Exascale computing technology challenges, in: High Performance Computing for Computational Science - VECPAR 2010, Springer Berlin Heidelberg, Berlin, Heidelberg, ISBN: 978-3-642-19328-6, 2011, pp. 1-25, http://dx.doi.org/10.1007/978-3-642-19328-6_1.

[5] A. Sedighi, Y. Deng, P. Zhang, Fairness of task scheduling in high performance computing environments, Scalable Comput.: Pract. Exp. 15 (2014) 273-285, http://dx.doi.org/10.12694/scpe.v15i3.1020.

[6] G. Rauchecker, G. Schryen, High-performance computing for scheduling decision support: A parallel depth-first search heuristic, 2015.

[7] D. Trystram, G. Mounié, P.-F. Dutot, Scheduling parallel tasks: Approximation algorithms, 2003, pp. 1-40.

[8] J. Hungershofer, On the combined scheduling of malleable and rigid jobs, IEEE (2004) http://dx.doi.org/10.1109/SBAC-PAD.2004.27.

[9] H. Casanova, F. Desprez, F. Suter, Minimizing stretch and makespan of multiple parallel task graphs via malleable allocations, 2010, pp. 1-10, http://dx.doi.org/ 10.1109/ICPP.2010.16.

[10] T. Guérouta, Y. Gaouaa, C. Artiguesa, G. Da Costa, P. Lopeza, T. Monteila, Mixed integer linear programming for quality of service optimizationin clouds, 2017, pp. 1-18, http://dx.doi.org/10.1016/j.future.2016.12.034. 
[11] P.-F. Dutot, M. Mercier, M. Poquet, O. Richard, Batsim: A Realistic LanguageIndependent Resources and Jobs Management Systems Simulator, ISBN: 978-3319-61755-8, 2017, pp. 178-197, http://dx.doi.org/10.1007/978-3-319-61756$5 \_10$.

[12] H. Casanova, A. Legrand, M. Quinson, Simgrid: A generic framework for largescale distributed experiments, in: Tenth International Conference on Computer Modeling and Simulation (Uksim 2008), IEEE, 2008, pp. 126-131.

[13] G.S. Prasanna, B.R. Musicus, Generalized multiprocessor scheduling and applications to matrix computations, IEEE Trans. Parallel Distrib. Syst. 7 (6) (1996) 650-664.

[14] M. Poquet, Simulation Approach for Resource Management, (Data structures and algorithms [cs.ds]), Université Grenoble Alpes, 2017.

[15] H.S. Azad, Advances in GPU Research and Practice, first ed., Morgan Kaufmann Publishers Inc., San Francisco, CA, USA, ISBN: 0128037385, 2016.
[16] P.E. Black, Greedy algorithm, 2005, https://www.nist.gov/dads/HTML greedyalgo.html.

[17] D. Bratton, J. Kennedy, Defining a Standard for Particle Swarm Optimization, in: 2007 IEEE Swarm Intelligence Symposium, 2007, pp. 120-127.

[18] M. Clerc, J. Kennedy, The particle swarm - explosion, stability, and convergence in a multidimensional complex space, IEEE Trans. Evol. Comput. 6 (1) (2002) 58-73.

[19] S. Helwig, J. Branke, S. Mostaghim, Experimental analysis of bound handling techniques in particle swarm optimization, IEEE Trans. Evol. Comput. 17 (2013) 259-271, http://dx.doi.org/10.1109/TEVC.2012.2189404.

[20] G. Da Costa, L. Grange, I. Courchelle, Modeling and Generating Large-Scale Google-Like Workload, 2016, pp. 1-7, http://dx.doi.org/10.1109/IGCC.2016. 7892623.

[21] S. García, F. Herrera, An extension on "statistical comparisons of classifiers over multiple data sets" for all pairwise comparisons, J. Mach. Learn. Res. 9 (2008). 